\title{
Gender Differences in Anatomic Variations of the Sternum Assessed by Multidetector CT Scan
}

\author{
Noor Kathem ${ }^{1 *}$ (D), Noor Abbas Hummadi Fayadh ${ }^{1}$ (D), Ahmed Al-Ali ${ }^{2}$, Hussein Al-Siraj ${ }^{2}$ (D) \\ ${ }^{1}$ Department of Surgery, College of Medicine, Al-Nahrain University, Baghdad, Iraq; ${ }^{2}$ Medical Trainee, College of Medicine, \\ Al-Nahrain University, Baghdad, Iraq
}

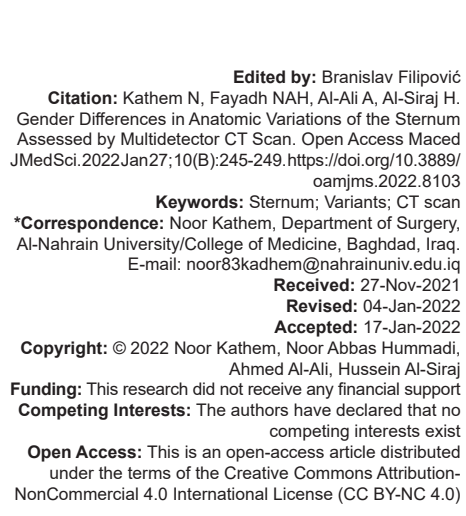

\section{Introduction}

The sternum is a key structure of the anterior chest wall. It is composed of 3 parts from superior to inferior: the manubrium, the sternal body, and the xiphoid process [1]. During embryologic development, the sternum is formed by endochondral ossification from about the $5^{\text {th }}$ month of life till shortly before birth. The manubrial ossification centers fuse before birth. The body of the sternum is formed from four sternal segments. The ossification centers of each segment fuse together around adolescence. The sternal segments fusion is usually completed by 25 years [2]. Several anatomical variations affecting the sternum have been reported. Variations can affect any of the three components but they are most pronounced in the xiphoid process [3]. Though most of these variations are asymptomatic, they can be confused with pathology [4], [5]; moreover, certain variants such as the sternal body foramina and clefts can pose particular difficulties when surgical interventions through the sternum are planned as in bone marrow aspiration [6], [7] or during acupuncture procedures [8]. Clefts and foramina can result from incomplete fusion of the sternebrae [9]. Episternal accessory bones have been described which could be fused with the manubrium and termed episternal tubercle or separated from it and termed episternal ossicle [7]. Variations affecting the xiphoid are many, it is variable in length and shape. It can have one, two, or even three-pointed endings. The distal end can be elongated or ventrally deviated which may feel like epigastric mass on clinical examination [3], [4], [5] One or more foramina may be seen in the xiphoid process from incomplete fusion of the ossification centers. The earliest studies addressing anatomic variations of the sternum relied on autopsy findings and plain radiographs. After the introduction of CT scanners and their increased use in clinical practice, several studies have been published describing the sternal anatomic variants utilizing the CT scanners with their multiplanar capabilities and 3D reconstruction [3], [10], [11], [12]. This study aims to assess certain anatomic variations of the sternum in adults and to address the gender differences of these variations using multidetector CT scan. 


\section{Patients and Methods}

\section{Patient sample}

This analytic cross-sectional study included patients referred for chest CT scan examination during 12 month period from January 2020 to January 2021. The indications of chest CT examination included evaluation of disease extent in COVID 19 patients, staging of known malignancy, cases of suspected pulmonary embolism, evaluation of COPD cases among other indications. Excluded from the study were patients with previous surgical interventions on the sternum including sternotomy, patient with chest wall deformities, and patient with a history of trauma to the sternum. Patients $<18$ years were also excluded from the study.

\section{CT protocol and image analysis}

The CT scan studies were performed on 2 types of scanners both 64-row multidetector scanners (Ingenia, Philips Medical Systems, Best, Netherland) and (Somatom definition AS, Siemens Healthineer, Erlangen, Germany) with slice thickness of 1-mm.

Image reconstruction and analysis were performed on workstation including 3D volume rendering, maximum intensity projection, multiplanar reconstruction (MPR), and curved MPR.

The images were evaluated by two specialist radiologists for the presence of sternal variations affecting the manubrium, the body, or the xiphoid process. The variants included:

1. Suprasternal accessory bone.

2. Manubrial variants (foramina, cleft, or sclerotic bands).

3. Sternal shape (flat, oval, or O-shaped).

4. Sternal body variants (foramina, sclerotic bands, or clefts).

5. Xiphoid process:

a. The shape (elongated, ventrally deviated S-shaped ...),

b. The xiphoid ending (single, double, or triple)

c. Xiphoid foramina and pseuoforamina

d. Extent of xiphoid ossification.

\section{Statistical analysis}

Data analysis and statistics were performed using the commercially available statistical package for social sciences program. Categorial variables were presented as numbers and frequencies. The unpaired t-test was used to evaluate statistical difference regarding various variants between men and women. A $p<0.05$ was considered statistically significant.

\section{Results}

The total number of patients included in the study was 817 with a mean age of $48.5 \pm$ 16.32 years and age range of $18-95$ years. $53.7 \%$ of the study population were men and $46.3 \%$ were women. The mean age of men in the study was 47 \pm 16.05 years while the mean age of women was 49 \pm 16.55 years.

\section{Suprasternal accessory bones}

Suprasternal accessory bone was present in $5.5 \%$ ( $n=45,17$ women and 28 men). Suprasternal tubercle was detected in $2.4 \%(n=20)$ of these cases $55 \%(11 / 20)$ were bilateral. Suprasternal ossicles were seen in $3.1 \%(n=26)$. Of these $53.8 \%(14 / 26)$ were bilateral. One case had a suprasternal tubercle on one side and a suprasternal ossicle on the other (Figure 1a).
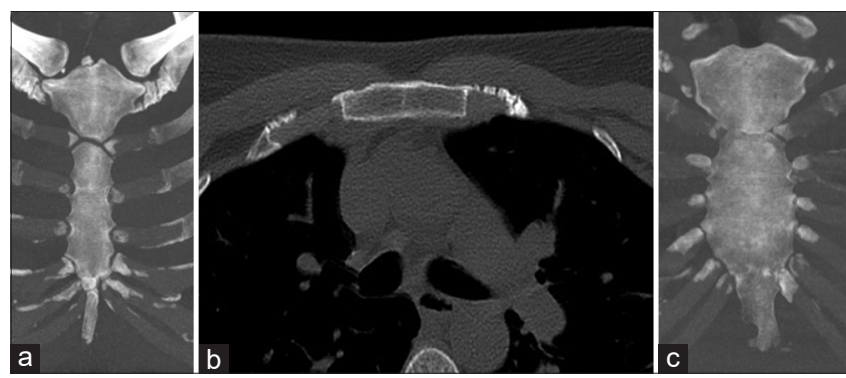

Figure 1: (a) maximum intensity projection image of a 39-year-old male patient showing bilateral sternal accessory bones, an ossicle on the right, and a tubercle on the left. (b) axial CT scan bone window showing midline sclerotic band in the manubrium. (c) O-shaped sternum.

\section{Manubrium}

Variations of the manubrium were detected in $1.5 \%$ of cases ( $n=12,4$ women and 8 men), these included manubrial foramina in $0.5 \%(n=4)$ and sclerotic bands in $1.1 \%(n=9)$ (Figure 1b). One patient had both sclerotic bands and a foramen.

\section{Sternum}

Sternal shape: The sternum was flat in most patients $(75.4 \%)(n=616,276$ women and 340 men), oval shaped in 23.5\% ( $\mathrm{n}=192,95$ women and 97 men) and O-shaped in 1.1\% $(n=9,7$ women and 2 men) (Figure 1c).

Sternal body variation: these were present in $7.7 \%$ of cases ( $n=63,33$ women and 30 men). Sternal foramina were seen in $4 \%$, sclerotic bands in $3.2 \%$ and clefts were rare detected in only $0.5 \%$.

\section{Xiphoid process}

Most of the anatomic variation detected 
involved the xiphoid process. The xiphoid bone was absent in $1.5 \%$ ( $n=12,9$ women and 3 men).

Regarding the pattern of xiphoid ossification, it was totally ossified in most patients $82.98 \%$. It was partially ossified superiorly in $14.41 \%$ of cases and partially ossified inferiorly in $1.99 \%$. No trace of xiphoid bone ossification was seen in 5 cases $(0.62 \%)$. The xiphoid ending was single in $65.71 \%$, double in $32.05 \%$ (Figure 2a), and triple in $2.24 \%$.

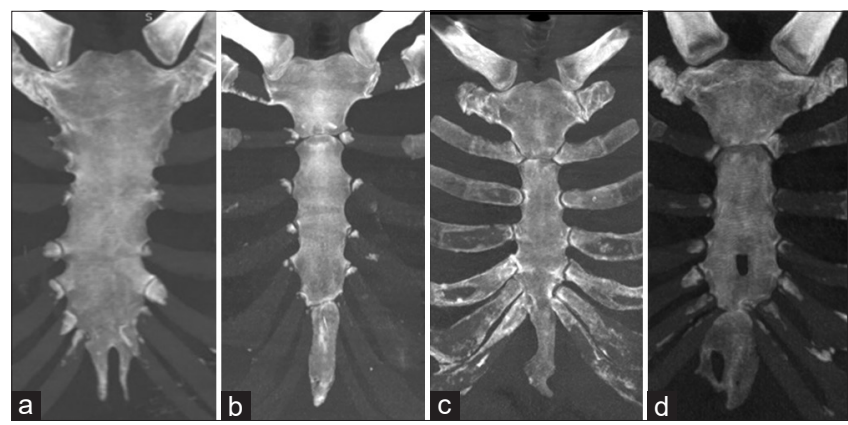

Figure 2: Coronal maximum intensity projection CT scan imaged showing (a) double-ended xiphoid process, (b) very long xiphoid process, (c) reversed S-shaped xiphoid, and (d) two xiphoid foramina and a sternal foramen

The xiphoid bone was elongated in $10.43 \%$ (Figure 2b), it was ventrally deviated in $16.77 \%$. In $10.06 \%$ of cases, it was both elongated and ventrally deviated. Reversed S-shaped xiphoid bone was detected in only 3 cases $(0.37 \%)$, all were women (Figure 2c).

Xiphoid foramina were seen in $17.39 \%$ of cases ( $n=140,37$ women and 103 men). Pseudo-foramina at the site of the sternoxiphoid junction were seen in 3.6 $\%$ ( $n=29,17$ women and 12 men). In 2.8\% ( $n=23$, 7 women and 16 men) multiple xiphoid foramina were seen (2, 3 or more) (Figure $2 \mathrm{~d}$ ).

\section{Comparison of the gender difference in the frequency of sternal variations}

\section{Suprasternal accessory bone}

No significant difference in the frequency of accessory suprasternal bone between men (6.4\%) and women $(4.5 \%)$ with a $p$ value of 0.762

\section{Manubrial variations}

No significant gender difference was seen regarding anatomic variations of the manubrium which were detected in 4 women $(1.1 \%)$ and 8 men (1.8\%). These variations were as shown in Table 1 . In the study population, no manubrial clefts were detected. One woman had both a sclerotic band and a foramen.
Table 1: Variations of the manubrial variations in women and men

\begin{tabular}{llll}
\hline Manubrial variation & Women & Men & p-value \\
\hline Cleft & $0(0.0 \%)$ & $0(0.0 \%)$ & 1.000 \\
Sclerotic band & $3(0.8 \%)$ & $6(1.4 \%)$ & 0.516 \\
Foramen & $2(0.5 \%)$ & $2(0.5 \%)$ & 1.000 \\
\hline
\end{tabular}

\section{Variations of the body of the sternum}

No significant difference in the frequency of anatomic variations of the sternal body between women and men which were detected in $8.7 \%$ of women $(\mathrm{n}=$ 33 ) and $6.8 \%$ of men $(n=30)$. Most of the cases of O-shaped sternum were seen in females (7 out of 9 ) (Table 2).

Table 2: Variations of the body of sternum in women and men

\begin{tabular}{llll}
\hline Variation & Women & Men & p-value \\
\hline Shape of the sternum & & & \\
$\quad$ Flat & $276(73 \%)$ & $340(77.4 \%)$ & 0.167 \\
Oval & $95(25.1 \%)$ & $97(22.1 \%)$ & \\
O shape & $7(1.9 \%)$ & $2(0.5 \%)$ & \\
Sternal body variation & $33(8.7 \%)$ & $30(6.8 \%)$ & 1.000 \\
Sternal cleft & $3(0.8 \%)$ & $1(0.2 \%)$ & 0.341 \\
Sternal sclerotic band & $16(4.2 \%)$ & $10(2.3 \%)$ & 0.161 \\
Sternal foramen & $14(3.7 \%)$ & $19(4.3 \%)$ & 0.723 \\
\hline
\end{tabular}

\section{Xiphoid process variations}

Significant difference in total xiphoid ossification was detected between men and women included in the AQ4 study which was significantly higher in men $(90.37 \%)$ than in women $(74.25 \%)$.

The ventrally deviated/elongated xiphoid process was observed in significantly higher percent of men $(33.25 \%)$ than women $(19.23 \%)$ with a $p<0.001$. Xiphoid foramina were detected in significantly higher number of men $(23.62 \%)$ than in women $(10.03 \%)$ with $p<0.001$ (Table 3).

Table 3: Variations of the xiphoid process in women and men

\begin{tabular}{llll}
\hline Variation & Women & Men & P-value \\
\hline Xiphoid ossification & & & \\
$\quad$ Absent & $4(1.08 \%)$ & $1(0.23 \%)$ & $*<0.001$ \\
$\quad$ Total & $274(74.25 \%)$ & $394(90.37 \%)$ & \\
$\quad \begin{array}{l}\text { Partial superior } \\
\quad \text { Partial inferior }\end{array}$ & $82(22.22 \%)$ & $34(7.8 \%)$ & \\
Xiphoidal ending & $9(2.44 \%)$ & $7(1.61 \%)$ & \\
$\quad$ Single & $253(68.56 \%)$ & $276(63.3 \%)$ & 0.287 \\
$\quad$ Double & $108(29.27 \%)$ & $150(34.4 \%)$ & \\
$\quad$ Triple & $8(2.17 \%)$ & $10(2.29 \%)$ & \\
Xiphoid shape & & & \\
$\quad$ Normal & $252(68.29 \%)$ & $243(55.73 \%)$ & $<0.001$ \\
$\quad$ Elongated & $41(11.11 \%)$ & $43(9.86 \%)$ & \\
$\quad$ Ventral deviation & $48(13.01 \%)$ & $87(19.95 \%)$ & \\
$\quad$ Elongated with ventral & $23(6.23 \%)$ & $58(13.3 \%)$ & \\
$\quad$ deviation & & & \\
$\quad$ C-shape & $2(0.54 \%)$ & $5(1.15 \%)$ & \\
$\quad$ Reverse S shape & $3(0.81 \%)$ & $0(0.0 \%)$ & \\
Xiphoid foramen & $37(10.03 \%)$ & $103(23.62 \%)$ & $<0.001$ \\
Pseudo foramen & $17(4.61 \%)$ & $12(2.75 \%)$ & \\
\hline${ }^{*}$ p-value $<=0.05$ & & & \\
\hline
\end{tabular}

\section{Discussion}

Several anatomic variations can affect the sternum, some have important clinical implications. 
During fetal life the sternum is formed by fusion of several separate ossification centers, abnormal or failure of fusion of these segments can give rise to variations such as foramina and clefts [2]. In most patients, these variants are asymptomatic and discovered incidentally during imaging. In certain situations, they can endanger the patients if not recognized. There are several reported cases of fatal cardiac tamponade after attempted bone marrow aspiration through a sternal foramen or during acupuncture [6], [7], [8]. To the best of our knowledge, no data till date exists regarding the gender difference in the frequency of anatomic variations of the sternum using multidetector CT scan.

The percent of accessory suprasternal bones in this study was $5.5 \%$, suprasternal ossicles seen in 3.1 $\%$ and suprasternal tubercle in $2.4 \%$. Relatively higher percent of accessory bones were detected in other studies reporting suprasternal ossicles in approximately $6.9 \%$ of subjects and tubercles in $0.01 \%$ [7]. In Yekeler et al. study [3], accessory bones were seen in $8.1 \%$, these were ossicles in $4.1 \%$ and tubercle in $4 \%$. In this study, the frequency of suprasternal accessory bones was $4.5 \%$ in women and $6.4 \%$ in men with no statistically significant difference.

The percent of manubrial variation in this study was $1.5 \%$, foramina in $0.5 \%$, and sclerotic bands in $1.1 \%$, with one patient having both foramen and band, no cleft was seen. In Yekeler et al. study [3], higher percent of manubrial bands (11.1\%) was observed, clefts were seen in $0.6 \%$. No manubrial foramina were detected in their study. Bayaroğulları et al. [11] reported one manubrial foramen in their study $(0.4 \%)$ which is comparable to our results. Leite et al. [12] also reported 1 manubrial foramen in a CT scan of a women. Their sample included 501 CT scans and 427 skeletons. No manubrial clefts were detected in the latter study. In the current study, there was no significant difference in the frequency of anatomic variations of the manubrium between men and women detected in $1.1 \%$ of women and $1.8 \%$ of men.

The most common shape of the sternum in this study was flat seen in $75.4 \%$. This was comparable to the study done by Bayaroğulları et al. [11] which showed that the flat type was detected in $68 \%$. In a cadaveric study by Selthofer et al. study [13], the standard shape of the sternum found in $2 / 3$ of the studied human sterna (dry bones) was longitudinal oval.

The assessment of the sternal shape in our study was based on visual assessment of 3D reconstructed images of the sternum whereas the study by Selthofer et al. relied on measurements of the thickness and width of the sternum at different levels.

The overall frequency of anatomic variants of the sternum in the study population was $7.7 \%$, of these foramina were the most frequently detected in $4 \%$, bands in $3.2 \%$, and clefts in $0.5 \%$. Yekeler et al. [3] showed sternal foramina in $4.5 \%$ a result comparable to ours, however, higher frequency of sclerotic bands was reported in their study $(17.8 \%)$. Sternal clefts were seen in $0.8 \%$ of the study population. Babinski et al. [9] reported sternal foramina in $16.6 \%$ of sterna, their study population included 180 dry bones and cadavers. In another study by Babinski et al. [14] evaluated sternal foramina on CT scans, the percent of sternal foramina was reported to be $10.5 \%$. In the present study, there were no significant anatomic variants of the frequency of the sternal variants between women and men (foramina seen in $3.7 \%$ women and $4.3 \%$ of men).

In this study, the xiphoid process was the most likely part of the sternum to exhibit anatomic variations. The xiphoid bone was absent in 12 patients $(1.5 \%)$ of these 9 were women and 3 were men with no statistically significant difference. Yekeler et al. [3] showed that the xiphoid bone was absent in $1.1 \%$ of their studied population. Akin et al. [10] study which included 500 patients evaluated by CT had no record of the absent xiphoid process.

Xiphoid ossification was total in $82.98 \%$ and partial in $16.4 \%$, no trace of ossification of the xiphoid bone was observed in 5 cases $(0.62 \%)$. In Akin et al. study total ossification was in $50.8 \%$ of cases and partial ossification in $48.6 \%$. Yekeler et al. reported total ossification in $70.3 \%$ and absent ossification in $2.3 \%$. This study showed significant gender difference in the extent of xiphoid ossification, significantly higher percent of total ossification was reported in men than in women on the other hand four of the five patients with absent ossification of the xiphoid were women. Xiphoid foramina were detected in $17.39 \%$ of cases in this study $(n=140 / 817)$. Of these cases, $16.43 \%$ (23/140) multiple (two or more) xiphoid foramina were seen. In Yekeler et al. study [3] the percent of xiphoid foramina was relatively high detected in $27.4 \%$ of cases. In Akin et al. study [10], the percent of xiphoid foramina was even higher detected in $43.2 \%$. Regarding gender difference in xiphoid foramina, there was a significant difference in their frequency between men and women, the percent was higher in men (23.62\%) as compared to women $(10.03 \%)$. The xiphoid ending was single in most cases $(65 \%)$ lower than that reported in Yekeler et al. study $(71 \%)$, triple-ended xiphoid was detected in $2.24 \%$, while in Yekeler et al. study, the ending was triple in significantly less patients $(0.7 \%)$. In Akin et al. study, single ending xiphoid was seen in $62.6 \%$, triple ending xiphoid was seen in $4.6 \%$ higher than in our study. There was no significant difference in the type of xiphoid ending between men and women included in the study. The xiphoid ending was ventrally deviated in $16.77 \%$ of cases. Akin et al. reported ventral deviation of the xiphoid in significantly higher percent $(65.4 \%)$. Bayaroğulları et al. also reported relatively higher frequency of ventral deviation of the xiphoid in $54 \%$. In this study, there was significant difference in the frequency of ventrally deviated and elongated/ventrally deviated xiphoid processes between men and women, the xiphoid process was ventrally deviated in $19.95 \%$ 
of men and $13.01 \%$ of women, the elongated ventrally deviated xiphoid was detected in $13.3 \%$ of men and $6.23 \%$ of women.

\section{Conclusion}

Some anatomic variants of the sternum are important to recognize and address as they can be misinterpreted as pathology or may pose a risk if interventions through the sternum are to be performed. Certain anatomic variants of the sternum are more likely to be encountered in males than in females namely, xiphoid foramina, ossification of the xiphoid, and ventral deviation of the xiphoid.

\section{Acknowledgment}

Our deep gratitude to Dr. Majid Hameed Ahmed, who performed the statistical work and gave advices for finalization of the manuscript writing.

\section{References}

1. Moore KL, Dalley AF, Agur AM. Clinically Oriented Anatomy $7^{\text {th }}$ ed. United States: Lippincott, Williams \& Wilkins; 2013.

2. Coward K, Wells D, editors. Textbook of Clinical Embryology. Cambridge: University Press; 2013.

3. Yekeler E, Tunaci M, Tunaci A, Dursun M, Acunas G. Frequency of sternal variations and anomalies evaluated by MDCT. AJR Am J Roentgenol. 2006;186(4):956-60. https://doi.org/10.2214/ AJR.04.1779 PMid:16554563

4. Lachkar S, Iwanaga J, Tubbs RS. An elongated dorsally curved xiphoid process. Anat Cell Biol. 2019;52(1):102-4. https://doi.

\section{org/10.5115/acb.2019.52.1.102 PMid:30984463}

5. Mashriqi F, D'Antoni AV, Tubbs RS. Xiphoid process variations A review with an extremely unusual case report. Cureus. 2017;9(8):e1613. https://doi.org/10.7759/cureus.1613 PMid:29098125

6. Fujita H, Nishimura S, Oyama K. Retrospective study on CT findings on the sternal bone after bone marrow aspiration procedure in hematological patients. Rinsho Ketsueki. 2009;50(12):1687-91.

PMid:20068275

7. Duraikannu C, Noronha OV, Sundarrajan P. MDCT evaluation of sternal variations: Pictorial essay. Indian J Radiol Imaging. 2016;26(2):185-94. https://doi.org/10.4103/0971-3026.184407 PMid:27413263

8. Chun KJ, Lee SG, Son BS, Kim DH. Life-threatening cardiac tamponade: A rare complication of acupuncture. J Cardiothorac Surg. 2014;9:61. https://doi.org/10.1186/1749-8090-9-61 PMid:24685234

9. Babinski MA, Rafael FA, Steil AD, Sousa-Rodrigues CF, Sgrott EA. de Paula RC. High prevalence of sternal foramen: Quantitative, anatomical analysis and its clinical implications in acupuncture practice. Int J Morphol. 2012;30(3):1042-9. https:// doi.org/10.4067/S0717-95022012000300045

10. Akin K, Kosehan D, Topcu A, Koktener A. Anatomic evaluation of the xiphoid process with 64-row multidetector computed tomography. Skeletal Radiol. 2011;40(4):447-52. https://doi. org/10.1007/s00256-010-1022-1 PMid:20721551

11. Bayaroğulları H, Yengil E, Davran R, Ağlagül E, Karazincir $S$, Balcı A. Evaluation of the postnatal development of the sternum and sternal variations using multidetector CT. Diagn Interv Radiol. 2014;20(1):82-9. https://doi.org/10.5152/dir.2013.13121 PMid:24100061

12. Leite VM, de Souza Plácido $C F$, Gusmão $C L$, Soriano EP, Almeida AC, Antunes AA, et al. Sternal variation: Anatomicalforensic analysis. Int Arch Med. 2020;13:1-12. https://doi. org/10.3823/2626

13. Selthofer R, Nikolić V, Mrcela T, Radić R, Leksan I, Rudez I, et al. Morphometric analysis of the sternum. Coll Antropol. 2006;30(1):43-7. PMid:16617574

14. Babinski MA, Lemos L, Babinski MS, Gonçalves MD. Frequency of sternal foramen evaluated by MDCT: A minor variation of great relevance. Surg Radiol Anat. 2015;37(3):287-91. https:// doi.org/10.1007/s00276-014-1339-x

PMid:25023390 